\title{
Unloading and reverse yielding of a finite cavity in a bounded cohesive-frictional medium
}

\author{
Jidong Zhao*, Gang Wang \\ Department of Civil and Environmental Engineering \\ The Hong Kong University of Science and Technology \\ Clear Water Bay, Kowloon, Hong Kong
}

\begin{abstract}
Cavity expansion in finite or infinite media has important practical implications in many engineering areas. In this study, we investigate the elastic unloading and reverse yielding behaviours of cylindrical and spherical cavities in a bounded cohesivefrictional medium. Of particular interest is the critical state when reverse yielding in the hollow cylinder/sphere is imminent in relation to the cavity pressure, the cavity dimensions, as well as the material properties. The critical pressure and optimal thickness that lead to strengthening of the hollow cylinder/sphere by so-called "overstrain" have been determined analytically. Both quantities are found to be explicit functions of the frictional angle of the material. The study considers the Mohr-Coulomb criterion and the bounded medium, which include both Tresca and purely frictional materials as special cases and, at the same time, can be readily extended to the case of an infinite medium. Finally, the results are applied to the interpretation of pressuremeter tests in soils, weak rocks and cemented sands.
\end{abstract}

Key words: Cavity expansion; cohesive-frictional materials; unloading; overstrain; reverse yielding; hollow cylinder/sphere; Mohr-Coulomb; Tresca; pressuremeter test; shakedown.

\section{Introduction}

Analysis of the expansion of cylindrical and spherical cavities in finite or infinite media provides useful information for the study of important practical

* Corresponding author. Tel.:+852 2358 8481; Fax: +852 23581534.

Email address: Jzhao@ust.hk (Jidong Zhao). 
problems in many engineering areas. Bishop et al. (1945) were among the first to apply the cavity expansion theory to the prediction of the hardness of metals from indentation tests (see also Hill, 1950). The analysis of cylindrically expanded cavities has long been employed in practical applications such as autofrettage of pressure vessels and gun barrels. In other areas such as geotechnical engineering, the theory of cavity expansion has been useful in the prediction of the bearing capacity of deep foundations and the pull-out capacity of earth anchors, the analysis of the stability and deformation of underground excavation and tunnelling, the study of borehole instability, and the interpretation of soil properties through various in situ soil testing methods, such as the cone penetration test (CPT) and pressuremeter tests (PMT). A systematic summary of the cavity expansion theory may be found either in the classic monograph by Hill (1950), or the more recent and complete book by $\mathrm{Yu}(2000)$ with an emphasis on geotechnical/civil engineering applications.

Of particular interest here is the behaviour of elastic unloading and reverse yielding of a previously plastically loaded hollow cylinder/sphere of a finite cohesive-frictional medium. During the process of elastic unloading, the contraction of the outer layers of the hollow cylinder/sphere compresses the inner layers and leaves the internal surface in a state of tangential compression. A subsequent application of pressure less than the original maximum value strains the hollow cylinder/sphere elastically. In this way, the hollow cylinder/sphere can be strengthened by an initial overstrain. However, due to the accumulation of residual stresses, the process of unloading could potentially stress an element in the hollow cylinder/sphere to the yield point in the reverse direction during the removal of the applied cavity pressure. Hill (1950) investigated the behaviour of a hollow sphere in Tresca materials unloaded from a partly plastic state, and determined the critical loading condition as well as the optimal thickness of the hollow sphere at which the reverse yielding was excluded in purely cohesive materials such as metals. A thorough investigation of a similar problem with cohesive-frictional materials could be useful in many other areas such as geotechnical engineering. For example, the selfboring pressuremeter has been developed as one of the best in-situ tests for geotechnical investigations, and the results of pressuremeter tests in soils are widely used to derive fundamental soil properties. Theoretical interpretation methods developed for pressuremeters are mostly based on the fundamental assumption that pressuremeter tests can be simulated as the expansion and/or contraction of an infinitely long, cylindrical cavity in soils, such that the cavity expansion theory can be conveniently employed in data interpretation. One of the important soil properties that can be deduced from pressuremeter tests in clay is its shear modulus. If the soil is assumed to behave as a linear elastic-perfectly plastic material, the shear modulus obtained from the unloading-reloading loop of the test is not dependent on the magnitude of the strain or pressure. As pointed out by Wroth (1982), it is important, however, to ensure that the unloading-reloading loops remain in the elastic region 
when conducting the loop tests. Analysis of the elastic unloading process to avoid reverse yielding is therefore of practical importance. Meanwhile, this study is useful in the shakedown analysis of a hollow cylinder/sphere as well. Shakedown is normally described as the phenomenon when a structure subjected to cyclic loads can deform plastically during the first few load cycles, but after that only elastic deformation occurs in the structure. The maximum load the structure can sustain for shakedown to occur is called the shakedown limit, which can be used as an important index for the design of structures subjected to cyclic loads. The loading-unloading behaviour and the resulting residual stress field can therefore provide useful information for shakedown analysis of an internally pressured hollow cylinder/sphere.

$\mathrm{Yu}(2000)$ summarised previous studies of cavity expansion theories by various researchers, e.g., cavity expansion in a Tresca material (Houlsby and Withers, 1988; Jefferies, 1988) and a Mohr-Coulomb material (Salencon, 1969; Houlsby et al., 1986; Withers et al., 1989; Senseny et al., 1989; Yu, 1990; Yu and Houlsby, 1995), as well as cavity contraction from an in-situ stress state in a Mohr-Coulomb material (Yu and Rowe, 1999). Despite these in-depth investigations, existing studies have been largely limited to the relatively simple case of infinite (or unbounded) media. It is surprising that there has been no detailed discussion of the unloading behaviour of a finite cavity in a bounded cohesive-frictional medium. A solution to this outstanding problem should yield more general results that can be readily reduced to special cases of infinite media for both Tresca and purely frictional materials. With such a general solution, we would be able to verify under what conditions the infinite case can be reasonably approximated by the finite solution. Such a solution, if available, can be a useful foundation for many practical problems, e.g., the interpretation of pressuremeter test data.

In this paper, we thoroughly investigate the elastic unloading and reverse yielding behaviours of a finite cylindrical or spherical cavity in a bounded MohrCoulomb material. First, we consider the stress distribution in the hollow cylinder/sphere with gradual relaxation of the internal cavity pressure. Then, we derive the critical pressure ratio between the internal cavity pressure and the outer pressure and the optimal thickness for the hollow cylinder/sphere, which together make strengthening by overstraining without reverse yielding possible. The effects of material properties are discussed. A minor error in Hill's (1950) classic work regarding the optimal thickness of a Tresca hollow sphere is also corrected. We show that the current study can recover results in infinite media as an extreme case, which will be demonstrated through comparison with the work by Wroth (1982) for the interpretation of pressuremeter results in clays and sands. This study serves as a useful supplement to cavity expansion theory, which can be used in practical applications such as pressuremeter tests and shakedown analysis. 


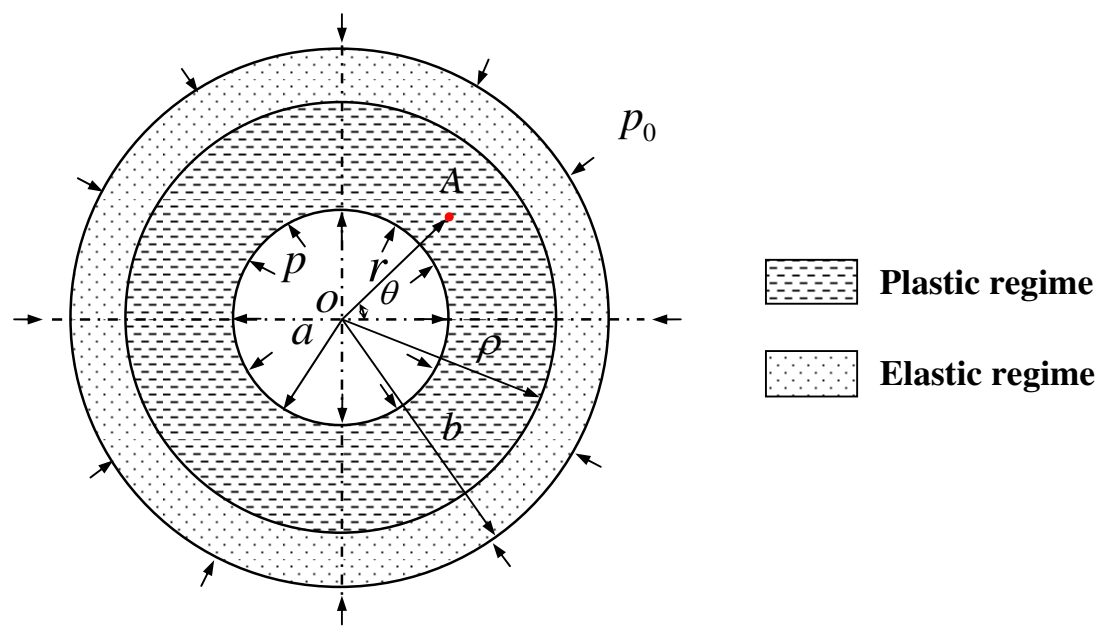

Fig. 1. Schematic of cylindrical/spherical cavity expansion in a finite cohesive-frictional medium. The cavity is denoted by the circle with a radius $a$; the outer surface radius is $b$. The plastic regime is denoted by an annulus with a radius of $\rho$. At the initial state a uniform pressure, $p_{0}$, is applied on the internal and external surfaces of the hollow cylinder/sphere. At the interim elastic plastic state, an increment of pressure, $\Delta p=p-p_{0}$, is applied to the internal surface of the cavity.

\section{Problem description}

A cylindrical/spherical cavity is assumed to be expanded in a bounded cohesivefrictional medium. The solid phase is in the shape of a hollow cylinder/sphere, and it is modelled as an isotropic elastic perfectly plastic material. The elastic behaviour of the material is governed by Hooke's law until the onset of yielding. The behaviour thereafter is governed by the Mohr-Coulomb criterion. As displacement analysis is not the focus of this paper, no assumption regarding the plastic flow rule is needed here. The hollow cylinder/sphere is assumed to be subjected to a uniform pressure, $p_{0}$, on both the internal and external surfaces, as shown in Figure 1. Note that in the case of self-boring pressuremeter tests, $p_{0}$ has a value comparable to the in-situ lateral stress, $\sigma_{h_{0}}$, in the soil, which can be measured from a typical pressure-expansion curve of the PMT test.

A cylindrical polar coordinate system $(r, \theta, z)$ is adopted for the cylindrical case, while a spherical coordinate system $(r, \theta, \varphi)$ is used for the spherical case. From this initial state, the pressure at the cavity surface is gradually increased from $p_{0}$ to $p$, which leads to an intermediate state as illustrated in Figure 1 . The current and initial radii of the cavity are denoted as $a$ and $a_{0}$ for the internal surface and as $b$ and $b_{0}$ for the external surface. Since this paper mainly deals with a state before the hollow cylinder/sphere enters a fully plastic state, the deformation can be generally regarded as small. In this case, we can safely assume that $a \cong a_{0}$ and $b \cong b_{0}$. However, as has been shown in numerous past studies (see, e.g., Yu, 2000), assumptions made about the 
deformation behaviour of the material affect the cavity expansion prediction. Early studies on cavity expansion always used a small strain assumption partly due to the simplicity and the usefulness in some particular applications, such as in the interpretation of pressuremeter tests where the strain is normally not greater than 10\% (Hughes et al., 1977). The assumption of large deformation was considered later in cavity expansion analysis in infinite media (e.g., Carter et al., 1986; Yu and Houlsby, 1991). In particular, it has been assumed that the total deformation might be large whereas the deformation in the elastic zone is infinitesimal. The limiting case of large deformations around a cylindrical cavity can be useful in the installation of driven piles in sands. In this paper, we do not attempt to seek closed-form solutions for the stresses and deformations in an entire space. We thus restrict our analysis up to the point of a fully plastic state with infinitesimal strain. Indeed, as suggested by Hill (1950), for cylinders with a not too large ratio of external and internal radii, $n=b_{0} / a_{0}$ (say, $n$ is less than 4 or 5), the strains and the displacements of the inner surface are relatively small so long as the fully plastic state has not been reached. Prior to this state, the variation in $a_{0}$ can be neglected when computing the stresses. While the large deformation solution remains interesting, it will be left for future study.

Tension is taken as positive in this paper. Following the classic cavity expansion theory, we simplify cylindrical expansion as a plane strain problem where $\sigma_{\theta \theta}$ and $\sigma_{r r}$ are, respectively, the maximum and minimum principal stresses (when $p$ is large enough to make it so). In the spherical case, the axisymmetric nature of the problem makes it simplier to have $\sigma_{\theta \theta}\left(=\sigma_{\varphi \varphi}\right)$ be the maximum principal stress and $\sigma_{r r}$ be the minor one. It is readily found that, in the absence of body forces, the equilibrium equation for the radial direction of the cavity can be expressed in the following unified form for both cases:

$$
\frac{d \sigma_{r r}}{d r}+\frac{k}{r}\left(\sigma_{r r}-\sigma_{\theta \theta}\right)=0
$$

where $k=1$ for the cylindrical case and $k=2$ for a spherical case.

The boundary conditions on the internal and external surfaces of the hollow cylinder/sphere take the following form:

$$
\left.\begin{array}{l}
T_{r}(a)=-\left.\sigma_{r r}\right|_{r=a}=p \\
T_{r}(b)=\left.\sigma_{r r}\right|_{r=b}=-p_{0}
\end{array}\right\},
$$

where $T_{r}$ is the surface traction.

The Mohr-Coulomb criterion governing the yielding behaviour of the cohesive- 
frictional material is (see also Chadwick, 1959; Carter et al., 1986):

$$
f=N \sigma_{\theta \theta}-\sigma_{r r}-2 c \sqrt{N}=0
$$

where $N=\frac{1+\sin \phi}{1-\sin \phi}$, with $\phi$ being the friction angle. $c$ is the cohesion of a material. Eq.(3) can be easily simplified to the case of purely frictional materials when the cohesion is set to zero $(c=0): N \sigma_{\theta \theta}-\sigma_{r r}=0$; or to the Tresca criterion by setting the frictional angle to $\phi=0$ (so that $N=1$ ): $\sigma_{\theta \theta}-\sigma_{r r}=2 c$. It is well known that the latter is a special case of importance to most metals and saturated soils, which exhibit no internal friction when deformed under undrained conditions.

\section{Elastic unloading, residual stresses and reverse yielding}

In this subsection, we present a unified closed-form solution for elastic unloading of a cylindrical/spherical cavity in a finite cohesive-frictional medium and determine the optimal thickness that leads to strengthening of the material by overstrain and the critical pressures below which reverse yielding can be avoided during the unloading. Following the procedure outlined by Hill (1950), if the hollow cylinder/sphere is unloaded from a partly plastic or fully plastic state, we can calculate the residual stresses (if a possible Bauschinger effect can be neglected) by subtracting the stresses of the current state from the elastic stresses.

The elastic stresses in a finite hollow cylinder/sphere are (c.f. Yu, 2000)

$$
\left.\begin{array}{l}
\sigma_{r r}=-p_{0}+\psi\left(p-p_{0}\right)\left[1-\left(\frac{b}{r}\right)^{(k+1)}\right], \\
\sigma_{\theta \theta}=-p_{0}+\psi\left(p-p_{0}\right)\left[1+\frac{1}{k}\left(\frac{b}{r}\right)^{(k+1)}\right]
\end{array}\right\} \quad(a \leq r \leq b)
$$

where

$$
\psi=\frac{a^{(k+1)}}{b^{(k+1)}-a^{(k+1)}} .
$$


In a partly plastic stage, the stresses in the elastic region are

$$
\left.\begin{array}{l}
\sigma_{r r}=-p_{0}+\xi\left[1-\left(\frac{b}{r}\right)^{(k+1)}\right], \\
\sigma_{\theta \theta}=-p_{0}+\xi\left[1+\frac{1}{k}\left(\frac{b}{r}\right)^{(k+1)}\right]
\end{array}\right\} \quad(\rho \leq r \leq b)
$$

whereas those in the plastic region are

$$
\left.\begin{array}{rl}
\sigma_{r r} & =\frac{2 c \sqrt{N}}{N-1}+\chi\left(\frac{\rho}{r}\right)^{\frac{k(N-1)}{N}}, \\
\sigma_{\theta \theta} & =\frac{2 c \sqrt{N}}{N-1}+\frac{\chi}{N}\left(\frac{\rho}{r}\right)^{\frac{k(N-1)}{N}}
\end{array}\right\} \quad(a \leq r \leq \rho)
$$

where

$$
\left\{\begin{array}{c}
\xi=\frac{2 c \sqrt{N}+(N-1) p_{0}}{(N-1)+\left(\frac{N}{k}+1\right)\left(\frac{b}{\rho}\right)^{(k+1)}} \\
\chi=-\frac{N(1+k) \xi}{(N-1) k}\left(\frac{b}{\rho}\right)^{(k+1)}
\end{array},\right.
$$

Since the cavity pressure, $p$, and the plastic radius, $\rho$, are not independent of each other, the following relation between the two can be found:

$$
p=-\frac{2 c \sqrt{N}}{N-1}-\chi\left(\frac{\rho}{a}\right)^{\frac{k(N-1)}{N}} .
$$

It is readily observed that the critical pressure for initial yielding $(\rho=a)$ is

$$
p_{a}=p_{0}+\frac{2 c \sqrt{N}+(N-1) p_{0}}{(N-1)+\left(\frac{N}{k}+1\right)\left(\frac{b}{a}\right)^{(k+1)}}\left[\left(\frac{b}{a}\right)^{(k+1)}-1\right]
$$

while the critical pressure at the fully plastic state $(\rho=b)$ is

$$
p_{b}=p_{0}+\left(p_{0}+\frac{2 c \sqrt{N}}{N-1}\right)\left[\left(\frac{b}{a}\right)^{\frac{k(N-1)}{N}}-1\right]
$$


We assume that the unloading is induced by a monotonical removal of the internal cavity pressure from its current value, $p$, to the value $p-\tilde{\lambda}\left(p-p_{0}\right)$, where the unloading factor is $0 \leq \tilde{\lambda} \leq 1$. Therefore, the change of pressure is $-\tilde{\lambda}\left(p-p_{0}\right)$, which is assumed to induce only elastic deformation in the material. To examine the possibility of material strengthening by overstrain as well as the condition to avoid reverse yielding during the removal of the applied load in the calculation of residual stresses, we superimpose the elastoplastic stresses at the initial pressure, $p$, in Eqs.(6) and (7) by the elastic stress (4) caused by this pressure difference:

For $\rho \leq r \leq b$,

$$
\left.\begin{array}{l}
\sigma_{r r}=-p_{0}+\xi\left[1-\left(\frac{b}{r}\right)^{(k+1)}\right]-\tilde{\lambda}\left(p-p_{0}\right) \psi\left[1-\left(\frac{b}{r}\right)^{(k+1)}\right], \\
\sigma_{\theta \theta}=-p_{0}+\xi\left[1+\frac{1}{k}\left(\frac{b}{r}\right)^{(k+1)}\right]-\tilde{\lambda}\left(p-p_{0}\right) \psi\left[1+\frac{1}{k}\left(\frac{b}{r}\right)^{(k+1)}\right]
\end{array}\right\}
$$

For $a \leq r \leq \rho$,

$$
\left.\begin{array}{c}
\sigma_{r r}=\frac{2 c \sqrt{N}}{N-1}+\chi\left(\frac{\rho}{r}\right)^{\frac{k(N-1)}{N}}-\tilde{\lambda}\left(p-p_{0}\right) \psi\left[1-\left(\frac{b}{r}\right)^{(k+1)}\right], \\
\sigma_{\theta \theta}=\frac{2 c \sqrt{N}}{N-1}+\frac{\chi}{N}\left(\frac{\rho}{r}\right)^{\frac{k(N-1)}{N}}-\tilde{\lambda}\left(p-p_{0}\right) \psi\left[1+\frac{1}{k}\left(\frac{b}{r}\right)^{(k+1)}\right]
\end{array}\right\}
$$

To check for reverse yielding, it follows from Eqs.(12) and (13) that

$$
\left.\begin{array}{l}
N \sigma_{r r}-\sigma_{\theta \theta}=(1-N) p_{0}+\xi\left[(N-1)-\left(N+\frac{1}{k}\right)\left(\frac{b}{r}\right)^{(k+1)}\right] \\
+\tilde{\lambda}\left(p-p_{0}\right) \psi\left[(1-N)+\left(N+\frac{1}{k}\right)\left(\frac{b}{r}\right)^{(k+1)}\right], \quad(\rho \leq r \leq b) \\
N \sigma_{r r}-\sigma_{\theta \theta}=2 c \sqrt{N}+\left(N-\frac{1}{N}\right) \chi\left(\frac{\rho}{r}\right)^{\frac{k(N-1)}{N}} \\
\quad+\tilde{\lambda}\left(p-p_{0}\right) \psi\left[(1-N)+\left(N+\frac{1}{k}\right)\left(\frac{b}{r}\right)^{(k+1)}\right], \quad(a \leq r \leq \rho)
\end{array}\right\}
$$

It is easy to verify that the numerical magnitude of $N \sigma_{r r}-\sigma_{\theta \theta}$ is greatest at 
the internal surface with the following unloading factor:

$$
\tilde{\lambda}^{\mathrm{r}}=\frac{\left(\frac{1}{N}-N\right) \chi\left(\frac{\rho}{a}\right)^{\frac{k(N-1)}{N}}}{\psi\left(p-p_{0}\right)\left[(1-N)+\left(N+\frac{1}{k}\right)\left(\frac{b}{a}\right)^{(k+1)}\right]}
$$

where the superscript $r$ denotes "reverse yielding". Utilizing Eqs.(8) and (10), Eq.(15) can be further written as:

$$
\tilde{\lambda}^{\mathrm{r}}=\left(\frac{p_{a}-p_{0}}{p-p_{0}}\right) \frac{(1+k)(1+N)\left[(N-1)+\left(\frac{N}{k}+1\right)\left(\frac{b}{a}\right)^{(k+1)}\right]\left(\frac{\rho}{a}\right)^{\frac{k(N-1)}{N}}}{k\left[(N-1)\left(\frac{\rho}{b}\right)^{(k+1)}+\left(\frac{N}{k}+1\right)\right]\left[(1-N)+\left(N+\frac{1}{k}\right)\left(\frac{b}{a}\right)^{(k+1)}\right]}
$$

When the plastic radius is $\rho$, no reverse yielding will occur during the unloading process when the unloading magnitude is less than the value presented in Eq. (16). It is also easy to verify that if the hollow cylinder/sphere is unloaded from the fully plastic state (i.e., $\rho=b$ ) to the initial pressure value $p_{0}$ (i.e., $\tilde{\lambda}^{r}=1$ ), the above equation gives the following maximum safe unloading pressure ratio:

$$
\left(\frac{p-p_{0}}{p_{a}-p_{0}}\right)_{\max }=\frac{(1+N)\left[(N-1)+\left(\frac{N}{k}+1\right)\left(\frac{b}{a}\right)^{(k+1)}\right]}{N\left[(1-N)+\left(N+\frac{1}{k}\right)\left(\frac{b}{a}\right)^{(k+1)}\right]}\left(\frac{b}{a}\right)^{\frac{k(N-1)}{N}}
$$

By using Eqs.(10), (11) and (16), the optimal thickness of a hollow cylinder/sphere can be given by the nontrivial solution to the following equation:

$$
\begin{array}{r}
N\left[(1-N)+\left(N+\frac{1}{k}\right)\left(\frac{b}{a}\right)^{(k+1)}\right]\left[1-\left(\frac{a}{b}\right)^{\frac{k(N-1)}{N}}\right] \\
=\left(N^{2}-1\right)\left[\left(\frac{b}{a}\right)^{(k+1)}-1\right] .
\end{array}
$$




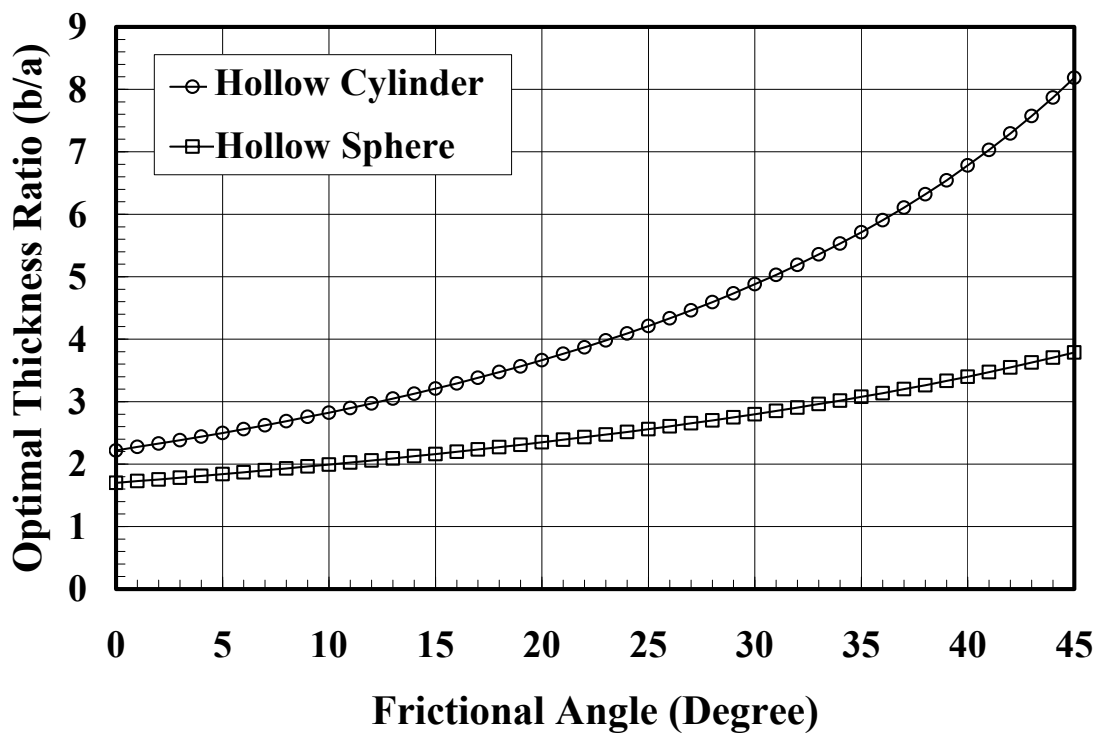

Fig. 2. optimal thickness ratios $b / a$ of a Mohr-Coulomb hollow cylinder/sphere at various internal frictional angles solved by Eq.(18).

\section{The optimal thickness of a hollow cylinder/sphere for overstrain}

The hollow cylinder/sphere cannot be too thin for it to be strengthened by overstrain. Eq.(18) gives the optimal thickness for a general Mohr-Coulomb material. It is interesting to first investigate the special case of a Tresca material where $N=1$. Applying a limit analysis in Eq.(18) leads to the following expression for the optimum thickness of a hollow cylinder/sphere in a Tresca medium:

$$
\ln \left(\frac{b}{a}\right)=\frac{2}{k+1}\left[1-\left(\frac{a}{b}\right)^{k+1}\right]
$$

The optimal thickness is independent of any material parameter. In the case of a Tresca hollow cylinder $(k=1)$, Eq.(19) gives an optimal thickness of $(b / a)_{\text {opt }}=2.22$, which implies that a Tresca hollow cylinder cannot be strengthened by overstrain if its outer radius is less than 2.22 times its inner cavity radius. It is also readily found that for the spherical case $(k=2)$, Eq.(19) presents an identical form to that obtained by Hill (1950, p.103). From this equation the optimal thickness of the hollow sphere is found to be $(b / a)_{\text {opt }}=1.70$. It is interesting to note that despite the fact that he expressed the correct form of Eq.(19) for the optimal thickness of a Tresca hollow sphere, Hill (1950) unfortunately provided an erroneous value of 4.92 as the solution in his classic monograph.

For a general Mohr-Coulomb hollow cylinder/sphere, the optimal thickness ratio, as can be readily observed from Eq.(18), is dependent upon the fric- 
tional property of the material only. By solving Eq.(18) using a numerical iteration scheme, the optimal thickness ratios of a hollow cylinder/sphere at various friction angles can be obtained, and they are shown in Figure 2. For both cylindrical and spherical cases, Figure 2 shows that the corresponding optimal thickness ratio increases with the friction angle of the material. The increase in the cylindrical case appears to be more significant, as compared with the spherical case. It is also observed that at the same friction angle, the corresponding optimal thickness ratio for the spherical case is always smaller than the cylindrical case. The difference between the two cases increases with the friction angle, and it can be as great as $116 \%$ of the smaller quantity. This observation implies that, in general, the frictional property makes it difficult to strengthen a material by overstrain, and the cylindrical case demonstrates a stronger trend in this direction than the spherical case because the stress field in the former case is relatively more inhomogeneous than in the latter case.

\section{Maximum safe unloading pressure to avoid reverse yielding}

Given the dimensions, the maximum safe pressure for a hollow cylinder/sphere to avoid reverse yielding during unloading can be easily determined from Eq.(17). It is interesting to have further discussion on the implications of the maximum safe unloading pressure.

\subsection{Safe pressure at optimal thickness}

If the hollow cylinder/sphere has an optimal thickness as defined in Eq.(18), it is readily found from Eq.(17) that the maximum safe pressure ratio is dependent on the frictional angle only. In connection with the results presented in Figure 2, the variation in the maximum safe pressure ratio is plotted against the internal frictional angle in Figure 3.

As can be seen from Figure 3, the cylindrical and spherical cases have identical curves for the safe pressure ratio with respect to the frictional angle. The maximum safe pressure ratio increases nonlinearly with the frictional angle of the soil. For $\phi=45^{\circ}$, this ratio could reach a value as high as 6.83 , in contrast with a much smaller value of 2 for a purely cohesive soil. In connection to Figure 2, however, we can see that this increase in the safe pressure threshold is achieved at a cost of a considerable increase in the thickness of the cylinder or sphere. This is more obvious for the case of a hollow cylinder. The safe pressure at $\phi=45^{\circ}$ is 2.14 times higher than that at $\phi=0$. To have such an increase in the safe pressure, a hollow cylinder has to have a thickness 3.69 


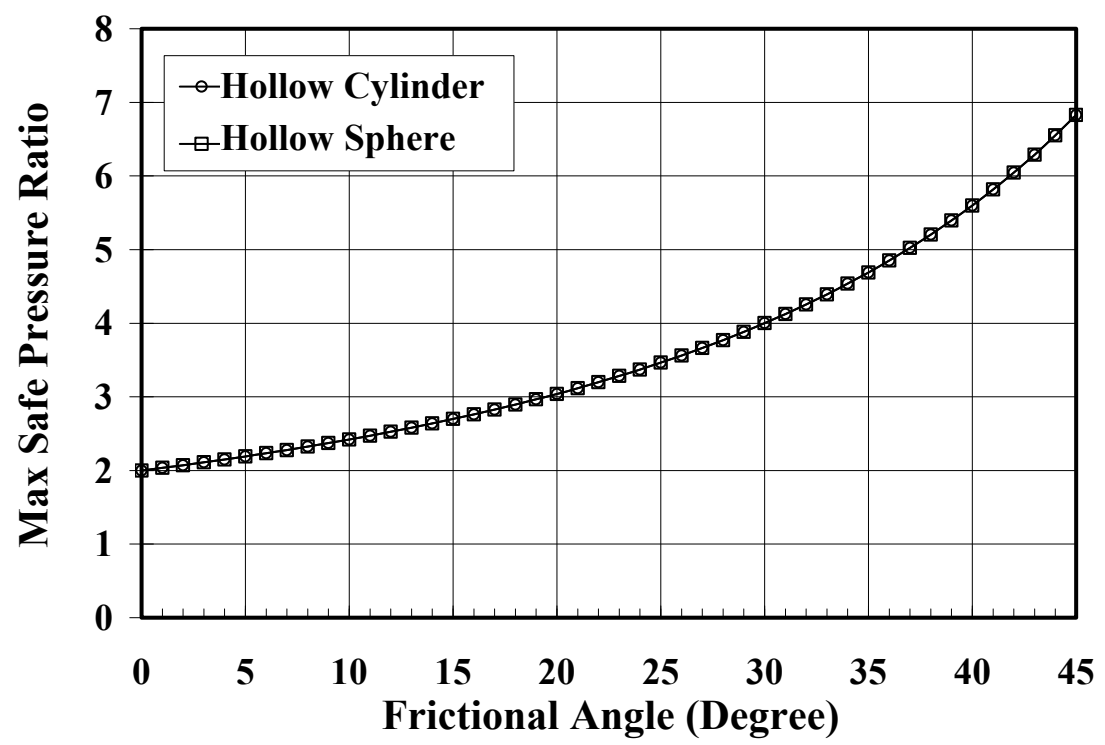

Fig. 3. Maximum safe pressure ratio $\left(\frac{p-p_{0}}{p_{a}-p_{0}}\right)_{\text {max }}$ for a Mohr-Coulomb hollow cylinder/sphere with optimal thickness as shown in Figure 2 to avoid reverse yielding during unloading.

times that of a purely cohesive hollow cylinder, as compared with a much smaller value of 2.23 in the spherical case.

\subsection{Purely cohesive solids $(\phi=0)$}

It is of particular interest to have a detailed discussion on the special case of purely cohesive solids where $\phi=0$ hence $N=1$ and therefore the Tresca criterion applies. In this case, the right-hand side of Eq.(17) gives a constant ratio of 2 for the safe pressure ratio:

$$
\left(\frac{p-p_{0}}{p_{a}-p_{0}}\right)_{\mathrm{m}}=2 \text {. }
$$

This result is consistent with that given by Hill (1950) for the case of a finite hollow Tresca sphere. We further note that the above result indeed holds universally for purely cohesive solids, regardless of the cavity types or the dimension of the host medium. Though the derivation has been started from a finite cavity in a finite medium, this safe pressure ratio has no dependence on the cavity size or the thickness of the hollow cylinder/sphere. We therefore expect that the same conclusion can be drawn for the case of finite cavity expansion in an infinite Tresca medium. Indeed, in an early study of unloading of a cylindrical cavity in an infinite Tresca soil, Wroth (1982) (see also Wroth (1984)) confirmed that the maximum cavity pressure reduction must be less 
than 2 times the undrained shear strength of the soil:

$$
(\Delta p)_{\max }=2 s_{u}
$$

where $s_{u}$ is the undrained shear strength of the soil $\left(s_{u}=c\right.$ here). Simple manipulation of Eq.(20) will prove that Wroth's finding is consistent with ours. Indeed, from the theory of cavity expansion (Yu, 2000), the initial yielding of a finite cavity in an infinite Tresca medium occurs at an pressure $p_{a}=p_{0}+c$. As such, $p_{a}-p_{0}=c=s_{u}$. Using this relation in Eq.(20) and setting $\Delta p=p-p_{0}$, we immediately observe that Eq.(20) leads to an identical form of Wroth's result in Eq.(21). While Wroth (1982) confined his results to the special case of a cylindrical cavity in an infinite Tresca medium, we can hereby extend his conclusion to a more general case as follows: for any finite cavity expanded in a Tresca medium, either bounded or unbounded, the maximum reduction of cavity pressure must not exceed 2 times the undrained cohesion of the soil, in order to ensure that the unloading-reloading process is purely elastic.

\subsection{The special case of an unbounded cohesive-frictional medium $(b=\infty)$}

We further note that the results developed in Section 3 can be easily degenerated to the case of a finite cavity in an infinite cohesive-frictional medium. In order for the results to be applicable to a finite cavity expanded in an infinite Mohr-Coulomb soil, we first recast Eq.(16) into the following form:

$$
\tilde{\lambda}^{\mathrm{r}}=\frac{\left(p_{a}-p_{0}\right)}{\left(p-p_{0}\right)} \frac{(1+k)(1+N)\left[(N-1)\left(\frac{a}{b}\right)^{(k+1)}+\left(\frac{N}{k}+1\right)\right]\left(\frac{\rho}{a}\right)^{\frac{k(N-1)}{N}}}{k\left[(N-1)\left(\frac{\rho}{b}\right)^{(k+1)}+\left(\frac{N}{k}+1\right)\right]\left[(1-N)\left(\frac{a}{b}\right)^{(k+1)}+\left(N+\frac{1}{k}\right)\right]}
$$

By setting $1 / b \rightarrow 0$, the above equation becomes

$$
\tilde{\lambda}^{\mathrm{r}}=\frac{\left(p_{a}-p_{0}\right)}{\left(p-p_{0}\right)} \frac{(1+k)(1+N)}{(k N+1)}\left(\frac{\rho}{a}\right)^{\frac{k(N-1)}{N}} .
$$

From Yu (2000),

$$
\left(\frac{\rho}{a}\right)^{\frac{k(N-1)}{N}}=\frac{(k+N)(2 c \sqrt{N}+(N-1) p)}{N(1+k)\left(2 c \sqrt{N}+(N-1) p_{0}\right)}
$$


Using Eq.(24) in Eq.(23) gives

$$
\tilde{\lambda}^{\mathrm{r}}=\frac{\left(p_{a}-p_{0}\right)}{\left(p-p_{0}\right)} \frac{(1+N)(k+N)}{N(k N+1)} \frac{(2 c \sqrt{N}+(N-1) p)}{\left(2 c \sqrt{N}+(N-1) p_{0}\right)}
$$

In the infinite case, by setting $1 / b \rightarrow 0$ again in Eq.(10), the critical pressure at initial yielding is obtained as:

$$
p_{a}-p_{0}=\frac{k\left(2 c \sqrt{N}+(N-1) p_{0}\right)}{k+N}
$$

Substitution of Eq.(26) into (25) leads to the following result:

$$
\tilde{\lambda}^{\mathrm{r}}=\frac{k(1+N)}{N(k N+1)} \frac{(2 c \sqrt{N}+(N-1) p)}{\left(p-p_{0}\right)}
$$

In an infinite Mohr-Coulomb medium when a finite cavity is expanded to a plastic state with plastic radius $\rho$ and then unloaded, Eq.(27) gives the safe unloading magnitude at which reverse yielding can be avoided. Note that for the case of a spherical cavity in an infinite Mohr-Coulomb medium where $k=2$, Eq.(27) is consistent with that obtained by Chadwick (1959) (Eq.(46) therein), except that $p$ in his equation is replaced by $p-p_{0}$ as we have assumed here that the monotonic unloading can reach a value of $p-\tilde{\lambda}\left(p-p_{0}\right)$ rather than $(1-\tilde{\lambda}) p$ as assumed by Chadwick (1959).

With a cohesionless material such as sand where $c=0$, Eq.(27) can be further simplified to

$$
\tilde{\lambda}^{\mathrm{r}}=\frac{k\left(N^{2}-1\right)}{N(k N+1)} \frac{p}{\left(p-p_{0}\right)}
$$

It is noteworthy that the above result covers another finding by Wroth (1982) regarding the maximum cavity pressure reduction in a cylindrical cavity in a infinite sand as a special case. Wroth (1982) expressed his results in terms of the effective stress quantities:

$$
\left(\Delta p^{\prime}\right)_{\max }=\frac{2 \sin \phi^{\prime}}{1+\sin \phi^{\prime}} p_{i n i}^{\prime}
$$

where $\left(\Delta p^{\prime}\right)_{\max }$ is the maximum reduction of the effective pressure allowed for elastic unloading of a cylindrical cavity in an infinite sand, $p_{i n i}^{\prime}$ is the effective cavity pressure at the start of pressuremeter unloading and $\phi^{\prime}$ is 
the drained angle of internal friction of the material. By simply letting $k=1$, $\Delta p=\tilde{\lambda}^{\mathrm{r}}\left(p-p_{0}\right)$ in Eq.(28), and meanwhile disregarding the difference between the effective stress and the total stress terms in Eq.(29) such that all the primes are dropped, it is readily found that these two equations become identical.

We would like to further illustrate Eq.(27) with a diagram by employing the idea of Wroth (1982). Figure 4 shows the theoretical limit of elastic unloading behaviour in pressuremeter test in an unbounded cohesive-frictional medium. Compared with Wroth (1982, p.160, Fig. 9) for the case of purely frictional sands, it is clear from Figure 4 that the cohesion of soils (which is the case at least for cemented sand, for example, if not for all in situ soils) can provide more room for the soil to be within the elastic limit if unloaded from a previously plastic state, which is typically experienced in pressuremeter test (if $k$ is set to be 1). This contribution could be significant when the pressure level (say, $\sigma_{r r}$ ) is relatively low. Wroth's (1982) results therefore provide a rather conservative estimate of the allowable amplitude of the stress cycle. It becomes less so, however, with the increase in $\sigma_{r r}$ and hence the mean stress level as the test progresses to later stages if the cohesion and frictional angle can be taken as constants of the soil. Meanwhile, it is also possible to construct a curve such as $T^{\prime} T T^{\prime \prime}$ as shown in Figure 4c, which serves as theoretical boundary to the elastic behaviour during unloading, for the general case of an unbounded cohesive-frictional soil. This is demonstrated in the following subsection for pressuremeter tests in weak rock and cemented sand.

\subsection{Application to weak rock and cemented sand}

Though it was originally developed for use in clays, the pressuremeter has also been used to test weak rocks and cemented sands (see, e.g., Haberfield, 1997). In weak rocks, the rate of the porewater pressure dissipation can be much quicker than in clays, although some rocks may have the same order of magnitude of permearbility as clays. In this case, undrained analysis can lead to significant errors in the estimation of the rock strength. As such, Haberfield (1997) suggested that a drained analysis method for weak rock or cemented sand is more appropriate in interpreting pressuremeter tests. For weak rocks or cemented sands under drained conditions, both cohesion and friction will contribute to the overall strength of the material, such that the general results in the preceding sections can be useful. Some of the pressuremeter test results obtained by Haberfield (1997) for a siltstone are used here as an example.

The test site Haberfield (1997) chose was located at the bottom of the Nubrik brick pit, in Scoresby, Australia. High capacity (20 MPa) pressuremeter tests were carried out at a depth of $2.5 \mathrm{~m}$ within a drilled borehole of $3 \mathrm{~m}$ deep. In his data fitting and drained analysis, the weak rock was assumed to be an 


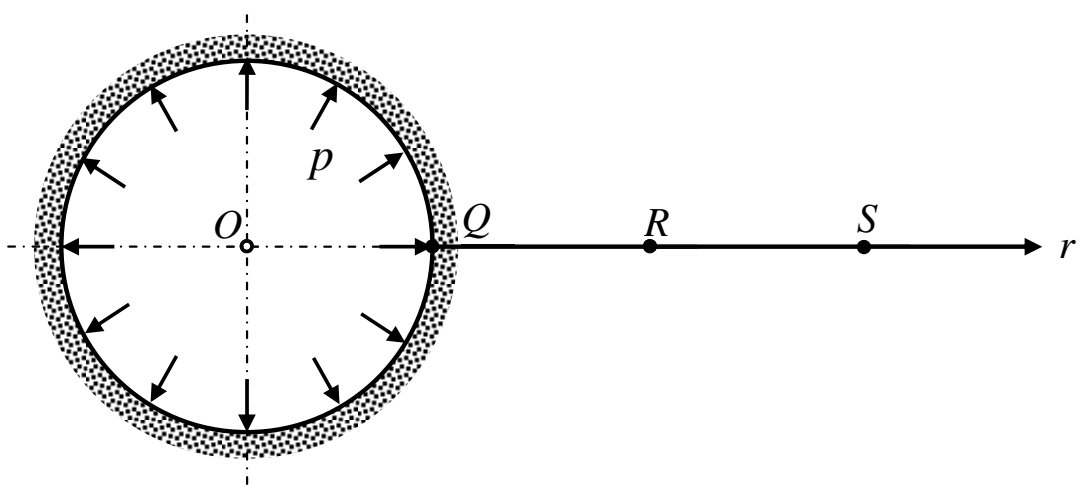

(a)

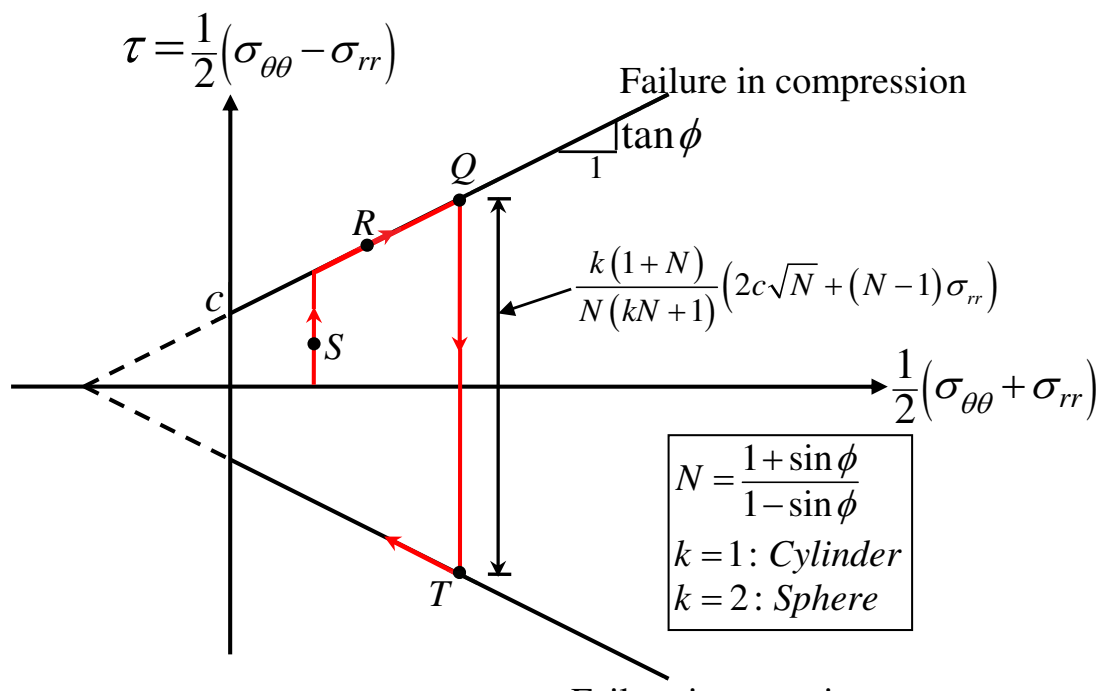

Failure in extension

(b)

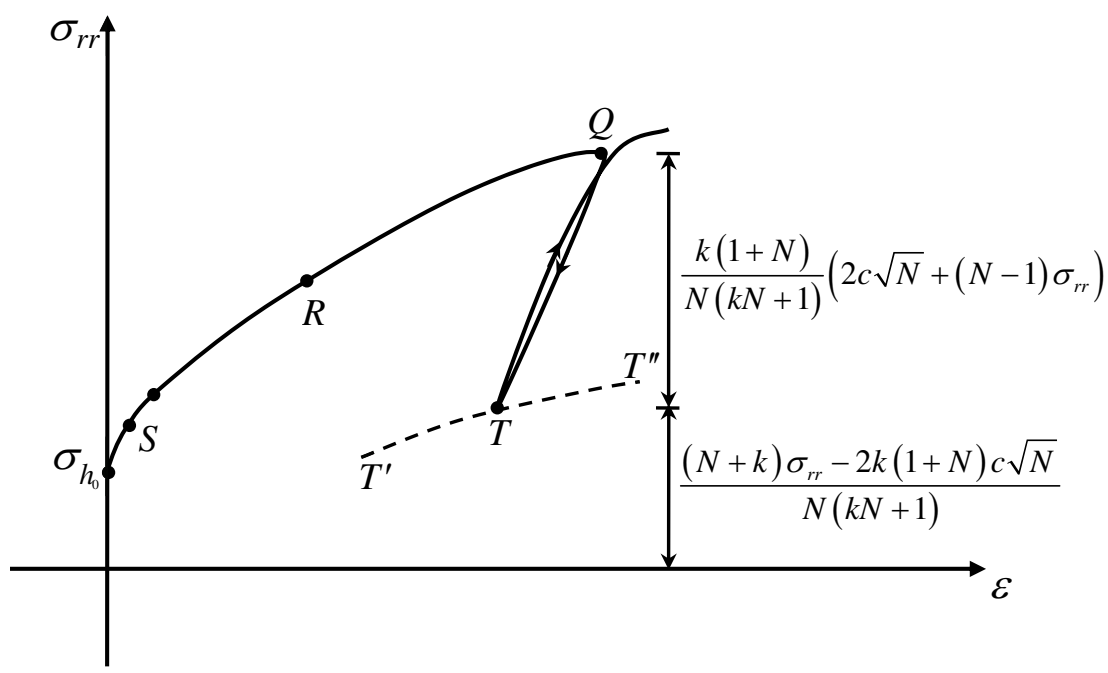

(c)

Fig. 4. The theoretical limit of the elastic behaviour of a cavity in an unbounded cohesive-frictional medium unloaded from a previously plastically loaded state (c.f., Wroth, 1982). 
elastic, perfectly plastic homogeneous and isotropic Mohr-Coulomb material. The following drained parameters were obtained from one of the pressuremeter tests labeled as PMT1(N-S): Secant Young's modulus, $E=1470 \mathrm{MPa}$; Poisson's ratio, $\nu=0.18$; Cohesion, $c=1.23 \mathrm{MPa}$; peak frictional angle, $\phi_{m}=44^{\circ}$; and residual frictional angle, $\phi_{r}=24^{\circ}$. The in situ horizontal stress was $p_{0}=0.2 \mathrm{MPa}$. He has also carried out an undrained analysis for comparison and obtained an undrained strength of $s_{u}=8.8 \mathrm{MPa}$ for PMT1(N-S).

The cavity expansion-pressure curve along with three unloading-reloading cycles is presented in Figure 5. In the same figure, we provide two unloading bound curves using Eq.(27) (also Figure 4c) by using the peak and residual frictional angle of the siltstone, respectively. Theoretically, a bigger unloadingreloading loop is preferred to provide more reliable interpretation of the soil/rock shear modulus than a smaller loop, and it offers extra flexibility in equipment control for a field test at the same time. We note that the use of the peak frictional angle offers a greater cushion than that created by the residual frictional angle. However, discretion has to be exercised here as the use of the peak frictional angle may overestimate the soil's strength in some cases.

In the meantime, if we use the undrained analysis result in conjunction with Eq.(20) or (21) for another prediction, the safe pressure reduction in this case will be $2 s_{u}=17.6 \mathrm{MPa}$. This means that at any point of the PMT curve in Figure 5, the cavity pressure can be totally unloaded to zero without causing reverse yielding, which is usually not in agreement with the test data. This again supports our observation that undrained analysis could lead to an overestimate of the rock's strength in this case, and a drained analysis is more appropriate for weak rocks/cemented sands.

\section{Conclusions}

A unified analytical solution has been derived for the problem of elastic unloading of a cylindrical/spherical cavity in a bounded cohesive-frictional medium that was previously loaded to a fully/partly plastic state. Major conclusions from the study are summarised as follows:

- The optimal thickness ratio (the external radius of the bounded medium over the cavity radius) required for the material to be strengthened by the process of overstrain depends only on the frictional angle of the material. For a Tresca material, this ratio remains a constant that is independent of the material parameters, the cavity types, or the bounded/unbounded nature of the host medium.

- The maximum safe pressure that the hollow cylinder/sphere can sustain to avoid reverse yielding in the process of unloading is a function of the 


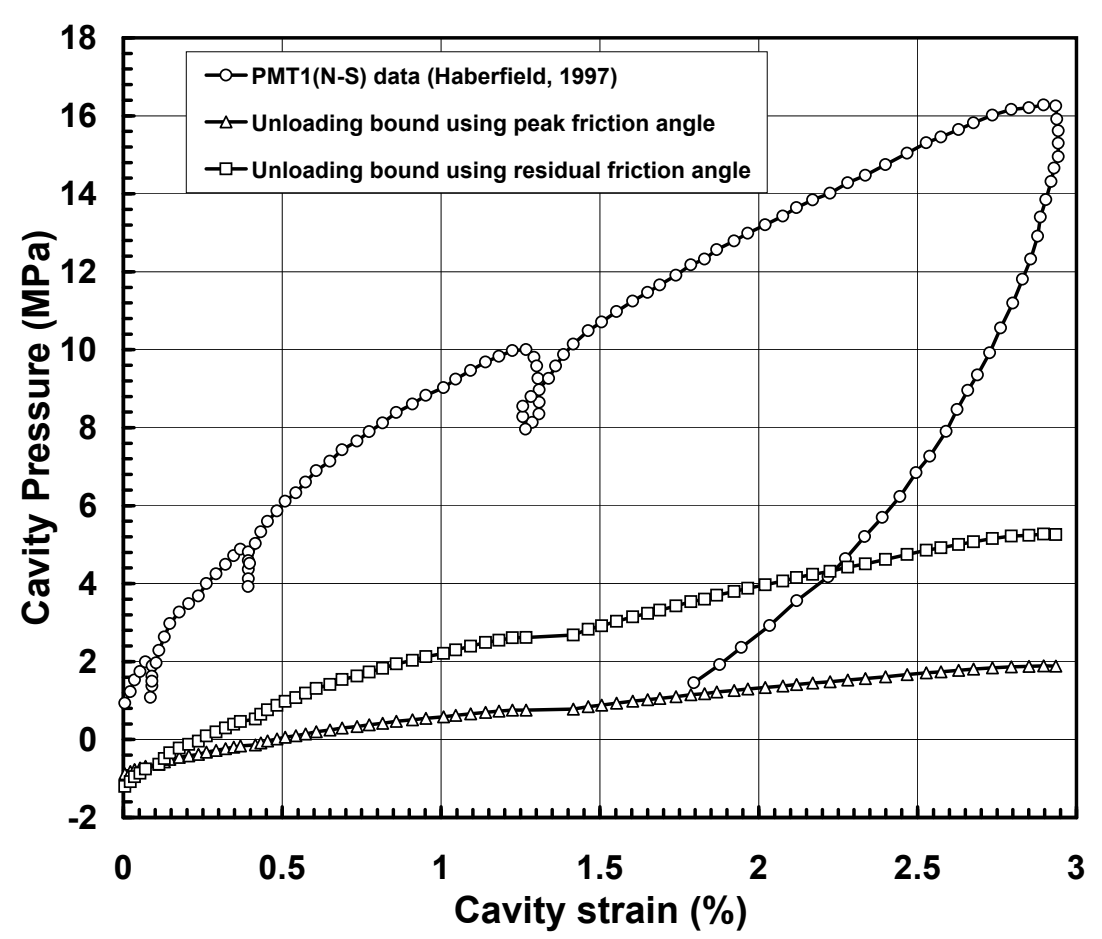

Fig. 5. Theoretical prediction of the bounding curve of unloading to avoid reverse yielding for pressuremeter tests in weak rock. The experimental data are from Haberfield (1997).

frictional angle, the cohesion of the material, as well as the dimensions of the hollow cylinder/sphere. If the hollow cylinder/sphere is at its optimal thickness, this safe pressure ratio is further found to depend on the frictional angle of the material only (note that such a pressure ratio is defined by Eq.(17) and the dependence of cohesion has been included in the expression of the critical pressure on initial yielding, as in Eq.(10)).

- The results obtained in this paper can be generalised to the case where the medium is unbounded, which is more useful for such practical applications such as the interpretation of pressuremeter tests. In particular, the current study has been shown to be able to include Wroth's (1982) solution as a special case.

- For a finite cavity (either cylindrical or spherical) expanded in a Tresca medium, the maximum reduction of cavity pressure must not exceed 2 times the (undrained) cohesion of the medium to ensure that the unloadingreloading process is purely elastic. This conclusion holds whether or not the medium is bounded. The conclusion is a more general finding than that drawn by Wroth (1982), which is only applicable to the special case of a cylindrical cavity in an unbounded medium.

- If both cohesive and frictional properties of the host medium can be characterized, which is typically the case for weak rocks or cemented sands, the safe allowable magnitude for the loading-unloading cycle can be greater than in cases where the material is assumed to be either purely cohesive (i.e., as 
in the case of metals or saturated clays) or purely frictional (i.e., as in the case of sands). The reverse loading threshold has been quantitatively determined in this paper for a general cohesive-frictional material (bounded or unbounded), with demonstrated application to pressuremeter tests in weak rocks.

This study contributes to the completeness of the current theory of cavity expansion and can be useful in practical applications such as in situ soil tests, shakedown analysis as well as indentation tests in material sciences.

\section{Acknowledgment}

This work was supported by RGC Direct Allocation Grants DAG08/09.EG04 and DAG08/09.EG13 of The Hong Kong University of Science and Technology and Grant No. 623609 from the Research Grant Council of Hong Kong.

\section{References}

Bishop, R.F., Hill, R., Mott, N.F., 1945. The theory of indentation and hardness tests. The Proceedings of the Physical Society, 57(3), 147-159.

Carter, J.P., Booker, J.R., Yeung, S.K., 1986. Cavity expansion in cohesive frictional soils. Géotechnique 36(3), 349-353.

Chadwick, P., 1959. The quasi-static expansion of a spherical cavity in metals and ideal soils. Q. J. Mech. Appl. Math., 12, 52-71.

Collins, I.F., Yu, H.S., 1996. Undrained cavity expansions in critical state soils. Int. J. Numer. Analy. Meth. Geomech. 20, 489-516.

Haberfield, C.M., 1997. Pressuremeter testing in weak rock and cemented sand. Proceeding of Institute for Civil Engineering - Geotechnical Engineering. 125, 168-178.

Hill, R., 1950. The Mathematical Theory of Plasticity. Oxford University Press, London.

Houlsby, G.T. and Withers, N.J., 1988. Analysis of the cone pressuremeter test in clay. Géotechnique, 38(4), 575-587.

Houlsby, G.T., Clarke, B.G., Wroth, C.P., 1986. Analysis of unloading of a pressuremeter in sand. Proceedings of the 2nd International Symposium on Pressuremeters, ASTM STP 950, 245-264.

Hughes, J.M.O., Wroth, C.P., Windle, D., 1977. Pressuremeter tests in sands. Géotechnique 27(4), 455-477.

Jefferies, M.G., 1988. Determination of horizontal geostatic stress in clay with self-bored pressuremeter. Canadian Geotechnical Journal, 25, 559-573.

Salencon, J., 1969. Contraction quasi-statique d'une cavite a symetrie 
spherique ou cylindrique dans un milieu elastoplastique. Annales des Ponts et Chaussees, 4, 231-236.

Senseny, P.E., Lindberg, H.E., Schwer, L.E., 1989. Elastic-plastic response of a circular hole to repeated loading. International Journal for Numerical and Analytical Methods in Geomechanics, 13, 459-476.

Withers, N.J., Howie, J., Hughes, J.M.O., Robertson, P.K., 1989. Performance and analysis of cone pressuremeter tests in sands. Géotechnique, 39, 433454.

Wroth, C.P., 1982. British experience with the self-boring pressuremeter. Proceedings of the Symposium on Pressuremeter and its Marine Applications, Paris, Editions Technip, 143-164.

Wroth, C.P., 1984. The interpretation of in situ soil tests. Géotechnique, 34(4), 449-489.

Yu, H.S., Houlsby, G.T., 1991. Finite cavity expansion in dilatant soils: loading analysis. Géotechnique 41(2), 173-183.

Yu, H.S. and Houlsby, G.T., 1995. Finite cavity expansion in dilatant soils: loading analysis. Géotechnique, 41(2), 173-183.

Yu, H.S. and Rowe, R.K., 1999. Plasticity solutions for soil behavior around contracting cavities and tunnels. International Journal for Numerical and Analytical Methods in Geomechanics, 23, 1245-1279.

$\mathrm{Yu}$, H.S., 1990. Cavity expansion theory and its application to the analysis of pressuremeters. PhD thesis, Oxford University.

Yu, H.S., 2000. Cavity Expansion Methods in Geomechanics. Kluwer Academic Publisher. 